\title{
Detecting Phase Shifts in Surface Plasmon Resonance: A Review
}

\author{
Y. H. Huang, ${ }^{1}$ H. P. Ho, ${ }^{1}$ S. Y. Wu, ${ }^{1}$ and S. K. Kong ${ }^{2}$ \\ ${ }^{1}$ Department of Electronic Engineering, The Chinese University of Hong Kong, Shatin, NT, Hong Kong \\ ${ }^{2}$ Programme of Biochemistry, School of Life Sciences, The Chinese University of Hong Kong, Shatin, NT, Hong Kong
}

Correspondence should be addressed to H. P. Ho, hpho@ee.cuhk.edu.hk

Received 8 May 2011; Accepted 11 June 2011

Academic Editor: Ken-Tye Yong

Copyright ( 2012 Y. H. Huang et al. This is an open access article distributed under the Creative Commons Attribution License, which permits unrestricted use, distribution, and reproduction in any medium, provided the original work is properly cited.

\begin{abstract}
Under certain conditions, a surface plasmon wave along a metal-dielectric interface can be excited by an optical beam. The reflected optical beam will then undergo changes in both intensity and phase. As the level of intensity or phase change is quite sensitive to the coupling conditions such as the molecule concentration on the metal surface, this phenomenon has been utilized for label-free detection of biological species and characterization of molecular interactions during the last two decades. Currently, most of the commercial surface plasmon resonance (SPR) sensors rely on the detection of absorption dip in angular or wavelength spectrum. However, recent researches have shown that phase detection has the potential to achieve lower limit of detection (LoD) and higher throughput. This paper, thus, intends to review various schemes and configurations for SPR phase detection. The performance advantages and disadvantages of various schemes will be emphasized. It is hoped that this paper will provide some insights to researchers interested in SPR sensing and help them to develop SPR sensors with better sensitivity and higher throughput.
\end{abstract}

\section{Introduction}

Surface plasmons are guided electron oscillations confined to a thin layer of the interface between two materials with negative and positive real parts of permittivity (e.g., a metaldielectric interface). Surface plasmons can be excited by photons when the optical wave vector parallel to the interface matches the propagation constant of surface plasmon. Under optimal conditions, optically excited surface plasmon resonance (SPR) could be quite strong and a large portion of optical energy is dissipated into a guided electromagnetic wave along the interface. As the extent of energy transfer is ultrasensitive to the coupling conditions, parameter such as the refractive index of dielectric layer can be accurately determined by monitoring the reflected light intensity or phase. This serves as the basis for various SPR sensors.

The first observation of surface plasmons is reported by Wood [1] when he used metallic grating to study the diffraction of polychromatic light and found an unexpected narrow dark band in the spectrum. The first theoretical investigation of surface electromagnetic waves was carried out by Zenneck [2] a few years later. Experimental researches on SPR, however, only started when Otto [3] and Kretschmann and Raether [4] demonstrated optical excitations of surface plasmons using different configurations of attenuated total reflection (ATR) method. These pioneering works had formed the foundation of SPR-based optical biosensors and stimulated numerous researches in the following decades. During the last two decades, the progress of semiconductor technologies and the high demands from biological research and pharmaceutical industry have boosted the rapid development of SPR-based sensors. Commercial SPR sensors are now available from numerous companies such as Biacore, AutoLab, Biosensing Instrument, ICx Nomadics (manufacturer of SPREETA previously under Texas Instruments), and Hofmann Sensorsysteme.

Most of current commercial SPR sensors, however, are based on the detection of reflectivity dip in the angular or wavelength spectrum. As the phase-shift in SPR is more prominent than its amplitude counterpart under optimal configuration, it can also been utilized to improve the sensitivity of SPR sensors [5, 6]. More importantly, phase detection-based SPR sensors are inherently more suitable for SPR imaging $[7,8]$ and have great potential to enable parallel detection of thousands of channels. They have received considerable research efforts in an accelerating manner in recently years.

Table 1 shows the typical performances of SPR sensors based on direct intensity measurement, angular interrogation, wavelength interrogation and phase detection. It can be seen that sensors based on phase detection offer better 
TABLE 1: Brief comparison between various schemes in SPR sensing.

\begin{tabular}{lcccc}
\hline SPR schemes & Intensity & Angular interrogation & Wavelength interrogation & Phase detection \\
\hline Resolution & $10^{-5} \mathrm{RIU}$ & $5 \times 10^{-7} \mathrm{RIU}$ & $10^{-6} \mathrm{RIU}$ & $4 \times 10^{-8}$ RIU \\
Dynamic range & $0.05 \mathrm{RIU}$ & $0.1 \mathrm{RIU}$ & $>0.1 \mathrm{RIU}$ & $5 \times 10^{-4}$ RIU \\
SPR imaging & Convenient & Difficult & Difficult & Convenient \\
\hline
\end{tabular}

sensitivity and convenient fulfillment of SPR imaging at the cost of narrower dynamic range.

In the literature, there are a few review articles [912] and books $[13,14]$ featured on SPR sensing and applications. However, so far no thorough reviews are dedicated to the detection of phase-shifts in SPR sensors. As phase measurement is more complicated than amplitudebased technologies and requires more know-how on optical components, modulation instruments, and image processing techniques, we feel it necessary to summarize phase detection techniques used in SPR sensors and to compare their advantages and disadvantages. This paper is, thus, dedicated to review recent works on the development of phase detection schemes in SPR sensors. The research papers are categorized into the following sections in terms of three major phase detection schemes, namely, optical heterodyne, polarimetry, and interferometry. It is hoped that this paper will facilitate researchers interested in this field and help them to develop proper phase sensitive SPR sensors for detecting smaller molecules with higher throughput.

\section{Sensors Based on Optical Heterodyne}

SPR sensors based on amplitude measurement are straightforward as photodetector measures the intensity directly. However, direct optical phase measurement is not as simple since no detectors are fast enough to resolve the oscillating intensity of an optical beam whose frequency is in the order of $10^{14}$. Thus, phase measurement normally relies on the interference between a signal beam and a reference beam by which a low-frequency signal can be generated and resolved by a photodetector. Optical heterodyne is one of the earliest techniques employed for phase measurement in SPR sensors. In this technique, two laser beams with slightly different frequencies are generated and made to interfere with each other which results in a signal with a "beat frequency" dependent on the frequency difference between the two original beams. By proper adjustment, this beat frequency will be low enough to be resolved by a photodetector and the phase difference between the two original beams can then be determined by comparing the beat signal to a reference signal with the same beat frequency using a commercial phase meter.

In 1996, Nelson and coworkers [15] reported a SPR phase sensor based on optical heterodyne detection. They used acousto-optic modulator (AOM) to generate two beams with frequency difference of $140 \mathrm{MHz}$ and split each beam into a reference part and a signal part by a polarizing beam splitter. The two reference parts are then combined with a linear polarizer and received by a photodetector. The signal is then low-pass filtered to obtain a wave with the beat frequency of $140 \mathrm{MHz}$, which is again mixed with a local oscillator at $140.01 \mathrm{MHz}$ to further reduce the frequency to $10 \mathrm{kHz}$ for convenient manipulation. The two signal parts go through the same procedures except passing through a SPR sensor with introduce a phase shift between the p- and scomponent. The comparison between the two $10 \mathrm{kHz}$ reference and signal waves using standard phase detection circuit has resulted in accurate determination of the SPR-induced phase shift. The heterodyne technique is insensitive to amplitude perturbation and environmental noise. Sensitivity as high as $5 \times 10^{-7} \mathrm{RIU}$ was predicted. The setup proposed was somewhat complicated due to hardware limitation, that is, the unavailability of instruments for proper frequency shifting and phase detection. Simpler setups for heterodyne detection had been proposed thereafter.

Instead of using AOM to generate the desired frequency shift, Shen and coworkers built a Zeeman laser based on a commercial He-Ne laser by applying high magnetic field to the laser cavity [16]. The frequency shift of the dual output components was further stabilized using feedback control and a frequency difference of $160 \mathrm{kHz}$ with stability of better than $3 \times 10^{-2}$ is achieved. In their work, both the phase jump and reflectivity change of the p-polarized wave through the SPR cell were measured using electronic phase meter and voltmeter. Experiments based on silver film were conducted and the results agreed well with theoretical prediction.

In 2001, Xinglong et al. built an immunosensor based on optical heterodyne and phase detection, where a Zeeman laser with frequency stability of $10^{-10}$ and frequency difference of $33.2 \mathrm{kHz}$ was used [17]. Reaction between ricin and its antibody was demonstrated and real time monitored by the proposed immunosensor. Phase change due to reaction was clearly observed and the maximum phase was found to be 8 degrees.

In 2003, Wu et al. included a total reflection device to their SPR sensor which helps to further enhance the phase shift between $\mathrm{p}$ - and s-components, and at the same time make the alignment of optical beams easier [18]. In their experiment (refer to Figure 1), two AOMs with driving frequency of 40 and $40.06 \mathrm{MHz}$ are employed which directly create a low frequency beat signal of $60 \mathrm{kHz}$ without further heterodyne as in Nelson's setup [15]. Phase difference between the signal arm and reference arm was then determined by a commercial phase meter. Experiments conducted to measure the phase response with varying incident angles for methanol, water, and ethanol have promised a refractive index resolution as high as $2 \times 10^{-7} \mathrm{RIU}$ for their system.

Since 2005, Chiu and coworkers have reported a series of research works on developing optical sensors based 


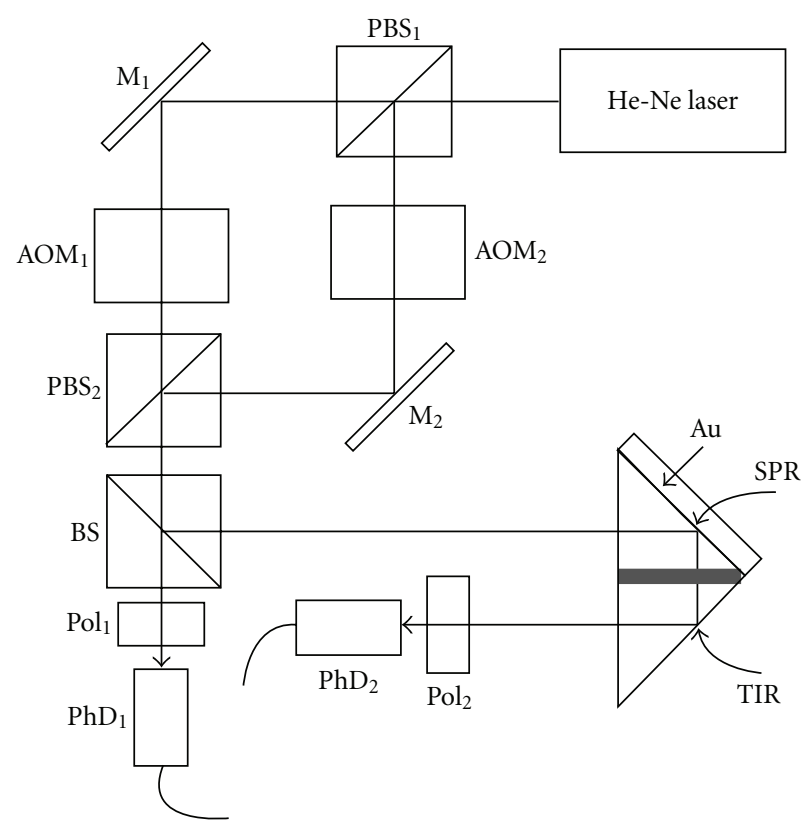

FIgURE 1: Schematic configuration of an experimental setup. PBS, polarizing beam splitter; $M$, mirror; BS, beam splitter; AOM, acousto-optomodulator; Pol, polarizer; $\mathrm{PhD}$, photodetector (Courtesy from [18]).

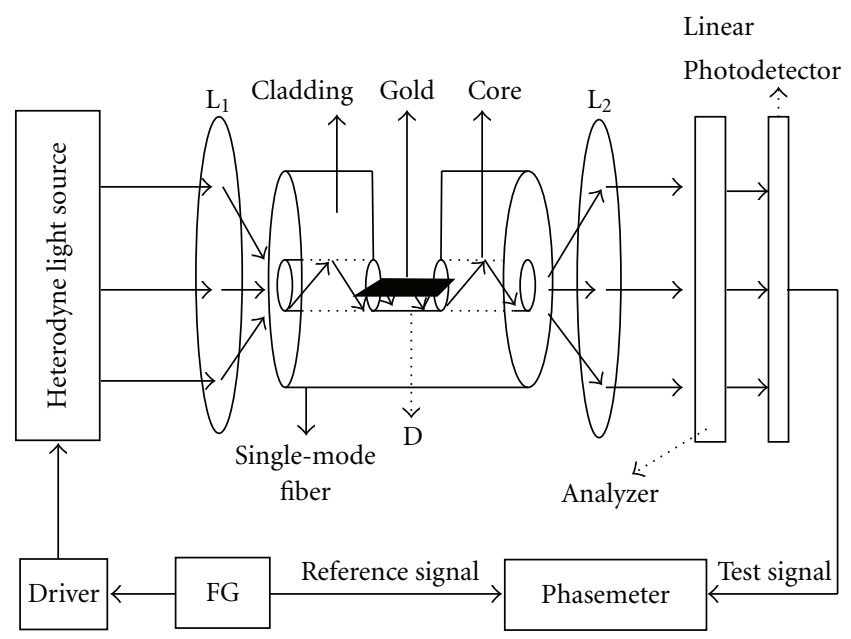

FIGURE 2: Schematic D-type optical fiber-based SPR sensor (Courtesy from [19]).

on heterodyne detection [19-22]. Instead of using prism coupler, their sensors were based on single mode optical fiber as shown in Figure 2. In the sensing area typically a few millimeters in length, the cladding and half of the fiber core was removed, the surface was then polished and coated with a thin layer of gold to form a D-type sensor. The sensing area was then immerged into the testing medium such as water, and the system was modeled as a modified Kretschmann configuration. Inside the optical fiber, incident light wave will undergo multiple attenuated total reflections and the SPR induced phase change will be greatly amplified.
However, the small numerical aperture of the single mode fiber has limited the incident angles from 86.5 to 89.5 degrees which is far from the resonance angle, thus only a medium resolution of $2 \times 10^{-6} \mathrm{RIU}$ was estimated. A big advantage of such optical fiber SPR sensor with multiple reflections over Kretschmann configuration is that the dynamic range has been greatly enhanced. Heterodyne light source had been used to pass through the optical fiber and the signal was compared to a reference signal by the function generator using a phase meter. Higher sensitivity than amplitude-based techniques had been obtained by this optical heterodyne detection method. Experiments conducted on alcohol with variable concentrations had shown good agreement between simulation and experiment results [19]. It should be noted that the total internal reflections from the cladding will also induce phase change in $\mathrm{p}$ - and s-polarized lights, but this can be ignored, as they are not affected by the refractive index change in the testing layer. Numerical simulations have also been carried out for both 3-layer (glass core-goldsensing layer) [20] and 4-layer (glass core-silicon dioxidegold-sensing layer) [21] configurations for their D-type optical fiber sensor, and optimal parameters were suggested. Interestingly, Wang [22] proposed a U-type optical fiber sensor for refractive index sensing recently. In this sensor, the cladding of the fiber is removed and the fiber core is immerged into the testing medium without any metal coating. Thus, the underlying principle is total internal reflection but not SPR. Fresnel's formulae are applied to estimate the phase change difference between the p- and s-components and optical heterodyne detection was used for sensing. Due to the over 70 total reflections, the sensor deliver a slightly better resolution of $1.6 \times 10^{-6}$ RIU.

In 2008, the highest refractive index resolution of $2.8 \times 10^{-9}$ RIU was reported by $\mathrm{Li}$ and coworkers [23]. Comparing to the previously reported heterodyne-based SPR sensor [17], where phase was measured by phase lock-in technique, active adjustment was employed by Li to equalize the amplitude of the reference and signal beams and the differential phase between them is subsequently retrieved by the amplitude modulation (AM) method which also help to realize real-time measurement. The equalization of signal and reference beam amplitudes, as well as the common path setup in optical heterodyne technique, has helped to eliminate noise from laser intensity fluctuation and resulted in ultra-high resolution. In their experiment, apparent phase change was observable by adding $0.00001 \%$ sucrose to pure water, which corresponding to refractive index change of $1.4 \times 10^{-8}$ RIU. As the signal magnitude is about 5 times of the noise level, the resolution of has been determined to be $2.8 \times 10^{-9}$ RIU. Experiments conducted on glycerinwater solution revealed a similar resolution of $7 \times 10^{-9} \mathrm{RIU}$, while that on interaction between mouse-IgG/antimouseIgG resulted in a sensitivity of $10 \mathrm{fg} / \mathrm{mL}$ ( $67 \mathrm{aM}$ ), which seems to be the highest ever achieved by SPR sensor.

Interestingly, before using phase detection for SPR sensing, the same research group had reported some works based on optical heterodyne technique $[24,25]$, but amplitude sensitive rather than phase sensitive method was used. In those works, they launched two pairs of p-and s-wave with 
slight frequency shift onto the SPR cell and heterodyne techniques were applied on the two p-components to produce the signal arm and at the same time to the two s-components to produce the reference arm. The sensor output was defined as the conditioned amplitude ratio between the signal arm and reference arm. By exciting two SP waves simultaneously, the sensor had been able to improve its sensitivity by 1-2 orders compared to conventional intensity-based technique, and refractive index resolution of $3.5 \times 10^{-7}$ RIU has been achieved based on an experiment on $0.001 \%$ sucrose-water solution. Compared to their recent result based on phase detection [23], it can be clearly observed that the sensitivity based on amplitude is 2 orders lower $\left(3.5 \times 10^{-7}\right.$ RIU vs. $2.8 \times 10^{-9}$ RIU) but the dynamic range is 3 orders higher $\left(10^{5}\right.$ vs. $10^{2}$ ). This could serve as a good experiment example for comparing phase and amplitude sensitive technique.

\section{Sensors Based on Polarimetry}

As the reflected light from a SPR cell is polarized and the ellipse of polarized light is directly related to phase change induced by SP wave, polarimetry (also called ellipsometry in this case), which measures the intensity ratio and phase difference between the $\mathrm{p}$ - and s-components of light, becomes a natural choice for SPR phase measurement.

The distinct feature of polarimetry for phase detection is the incorporation of a polarizer which helps to make the orthogonally polarized $\mathrm{p}$ - and s-components interfere at the photo sensor surface and results in a steady pattern. The interference pattern itself has reflected the phase difference between the $\mathrm{p}$ - and s-components (so-called polarization contrast); however, more accurate determination of phase difference depends on generating a series of interference patterns using various phase retarders or polarization modulators which help to provide abundant data for noise filtering and signal processing.

As early as in 1976, Abeles had figured out theoretical description of dependency of phase difference on the wave vector along the surface of a Kretschmann configuration and proposed the adoption of ellipsometry for investigating surface or interface reactions [5]. In 1990s, Herminghaus et al. used polarimetry technique with a Soleil-Babinet compensator to characterize thin films [26], while Kruchinin and Vlasov used a polarimetry setup to build a DNA-probe biosensor [27]. After 1998, a large number of research papers on SPR polarimetry emerged and helped to greatly enhance the sensitivity and imaging capability of SPR sensors.

Nikitin's research group experimentally measured the phase response of an SPR cell with varying incident angles using polarimetry setup [28, 29]. They also proposed a polarization phase contrast scheme to enhance the contrast of SPR intensity profile by orders of magnitudes, which resulted in higher sensor sensitivity and more flexibility in sensor configurations [30].

Ellipsometers with rotating analyzer have also been adopted for SPR sensing. Westphal and Bornmann employed commercial ellipsometer to measure both the amplitude ratio and phase change between the $\mathrm{p}$ - and s-components of light reflected from the SPR cell for biosensing [31].
From their bioaffinity experiments between streptavidin and antibodies, and hybridization experiments between DNA and complementary DNA strands, it turned out that the system yields a sufficient sensitivity for detecting biomolecular layers with effective thickness less than $10 \mathrm{pm}$. It was also found that phase response is significantly larger than the amplitude ratio response. Poksinski and Arwin used commercial ellipsometry-based SPR sensor for characterizing metal surface exposed to milk [32]. Abrupt phase changes have been observed during the processes of splashing and cleaning of milk on metal surface. Various metals had been investigated including gold, chromium, and iron, and different response curves were observed. This opens a door for potential applications of SPR sensors for automated monitoring in food industry. Naraoka and Kajikawa built an ellipsometer using rotating analyzer and sensitivity of $10^{-7}$ RIU had been reported [33].

In recent years, the method of differential surface plasmon ellipsometry, where the detected signal after the polarizer was compared to the modulation signal for phase detection has been extensively explored by Hooper and coworkers. In 2004, a Faraday rotator was employed to produce a sinusoidal variation of the polarization plane of the incident light. The output signal was then compared to the modulation signal by a phase detector to accurately determine the azimuth angle of the polarization ellipse of the reflected light from the SPR cell, and the change of azimuth angle was used to determine the refractive index change in the sensing layer [34]. Scheme with feedback control had also been proposed, where a feedback signal had been used to apply an additional quasistatic compensating voltage on the Faraday rotator in order to lock to zero in the differential signal. The resolution was found to be 2.5 times more sensitive with the feedback scheme and reached a refractive index resolution of $2 \times 10^{-7}$ RIU based on an experiment of mixing argon with nitrogen. In the same year, they reported similar phase sensing technique but with different polarization modulation equipment [35]. In that work, a liquid crystal (LC) polarization modulator was employed instead, as it is small, light-weight, low voltage, much cheaper and more importantly, it facilitates array sensing and SPR imaging. Same refractive index resolution of $2 \times 10^{-7}$ RIU had been obtained with the LC polarization modulator. In their following work [36], SPR imaging by use of the LC polarization modulator and high speed CMOS camera had been demonstrated. Differential signal for each pixel was obtained by temporal data processing of the captured image sequence and both the amplitude and phase information were subsequently retrieved. Effectiveness of the proposed phase imaging scheme had been demonstrated by an experiment, where the dielectric layer was exposed to ultraviolet radiation. The evolution of the phase information with regard to exposure time had been given and phase change had been clearly identified. The resolution of the method, however, was not available as the refractive index change due to exposure to ultraviolet radiation was not evenly distributed and could not been accurately identified.

Recently phase sensitive SPR sensing using polarimetry with photoelastic modulationtechnique has been extensively 


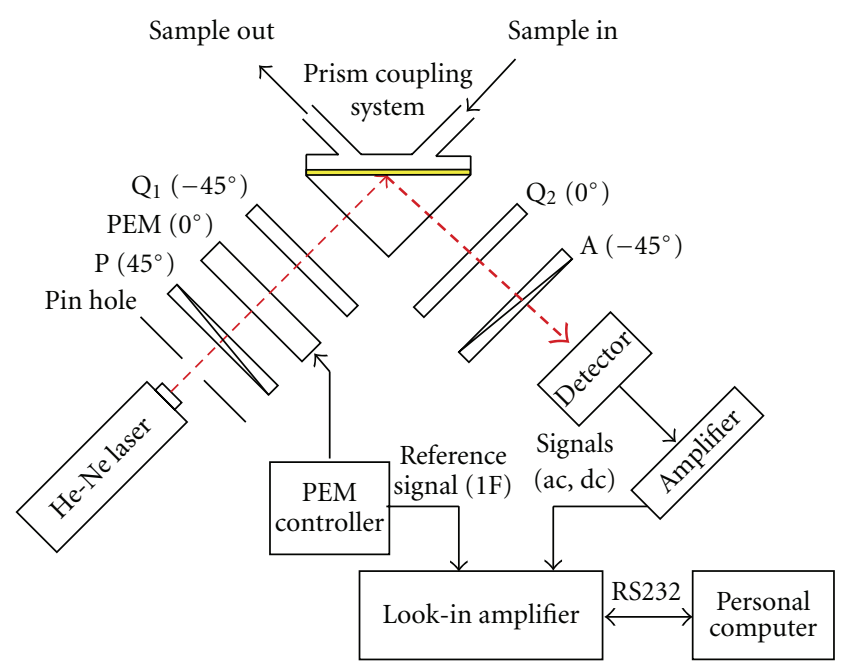

FIGURE 3: Schematic diagram of the SPR biosensor based on lowlevel birefringence phase detection (Courtesy from [37]).

investigated. Ho and coworkers had reported various configurations for phase measurement using photoelastic modulator since 2003 [37-39]. The photoelastic modulator was incorporated into the optical setup before or after the SPR cell to introduce a high frequency sinusoidal modulation of the phase retardation between the $\mathrm{p}$ - and s-components (A typical experiment setup is shown in Figure 3). The detected signal, which can be expanded by Bessel series, presents a series of harmonics due to the sinusoidal variation of retardation. By decomposing the detected signal using lock-in amplifier with input reference signals at the first and second harmonic frequencies of the photoelastic modulator, the amplitudes of first and second harmonics can be obtained and further processed to determine the phase retardation. This scheme was first used to determine the low-level birefringence [38], and later adopted for SPR biosensing [37]. Phase change with regard to varying concentration of glycerin in water had been demonstrated and the sensitivity was found to be $1.19 \times 10^{-6}$ RIU. The technique was also applied for real-time characterization of the binding reaction between bovine serum albumin (BSA) and anti-BSA biomolecules, and the detection limit was found to be $11.1 \mathrm{ng} / \mathrm{mL}$. By rearranging the optical geometry, a modified setup [39] had been proposed to determine the full polarization parameters including the azimuth angle, the intensity ratio of $\mathrm{p}$ - and s-component and the phase retardation of the polarized light reflected from the SPR cell. In particular, division of the first and second harmonics signal in this setup was found to represent the tangent of the double azimuth angle which provides a sensitive indicator of the refractive index change without compromising the dynamic range. This indicator is also less sensitive to stray light and laser intensity fluctuation and provides a better signal to noise ratio. As a result, a detection limit of $6 \times 10^{-7} \mathrm{RIU}$ or $15 \mathrm{ng} / \mathrm{mL}$ with a dynamic range in the order of $1 \times 10^{-2}$ RIU had been obtained. In 2008 , Stewart et al. reported an analytical investigation to optimize the photoelastic modulator based SPR polarimetry system and subsequently used it for biochemical sensing [40]. As a result, a refractive index resolution lower than $5 \times$ $10^{-7}$ RIU had been obtained and good repeatability had been demonstrated. Later, they demonstrated the multiplexing capability of their SPR polarimetry system and compared its performance with the commercially available SensiQ discovery system [41], and higher signal to noise ratio as well as lower detection limit had been obtained.

Kabashin and coworkers had investigated polarimetry based SPR sensing intensively in recent years [42-46]. In 2007, they used a photoelastic modulator for temporal modulation of the phase retardation. Instead of using the first and second harmonic components as in previous works [37], they used the second and third harmonic components [42] for SPR sensing which were found to provide a much better sensitivity than amplitude-based technique yet yielded a relatively wide dynamic range. As a result, detection limit of $2.89 \times 10^{-7}$ RIU with a dynamic range larger than $10^{-2} \mathrm{RIU}$ has been obtained. Similar method was then applied for the detection of streptavidin-BSA complex and a detection sensitivity of $1.3 \mathrm{nM}$ is reported [43], which was comparable to the best sensitivities reported in literature. SPR polarimetry using a spatial modulator had also been reported in 2008 where a wedge-shaped birefringence material was used to introduce a spatially distributed linear phase retardation over the imaging area [44]. The two-dimensional fringe patterns (the carrier fringes are induced by the birefringence wedge, while the distortion is induced by SPR phase distribution) were then captured by a CCD camera and processed by Fourier transform to extract the two-dimensional phase distribution. Phase change due to refractive index change of the SPR cell will then distort and move the original fringes, and the change amount can be obtained by simply subtracting the phase map obtained before and after the refractive index change. Even with relatively high noise level of 0.03 degree which was mainly due to laser power and temperature fluctuations and data insufficiency for averaging, a detection limit higher than $10^{-6}$ RIU had been achieved in calibration experiment. Experiment conducted on antibody binding to surface immobilized lysozyme had shown at least one order higher sensitivity of this phase scheme than amplitude scheme under the same setup. Besides the higher resolution compared to most amplitudebased techniques, this setup offers the additional advantage of very good spatial resolution which enables SPR imaging. SPR polarimetry using a mechanical modulation method was also proposed in 2008 [45]. In that work, a Wollaston prism was used to separate the s- and p-components and an optical chopper was then incorporated to introduce a high-frequency polarization modulation. Phase information at different harmonics of the modulation frequency was then extracted to determine the phase change caused by the bioreaction on the SPR cell. Phase measurement resolution as low as 0.007 degree and a refractive index detection limit of $3.2 \times 10^{-7}$ RIU has been demonstrated using this mechanical modulation method. In their recent work [46], a practical calibration method to determine the optimal incident angle for maximum phase response has been proposed. For a given SPR supporting film thickness, the angular SPR dip 
position in intensity curve may not coincide with the optimal incident angle for maximum phase response (except for the case of optimal film thickness), thus angular spectrum of intensity is not practical for determining the optimal incident angle. Simulation work revealed that angular spectrum of phase for different film thickness intersects at the optimal incident angle, which motivates a method for effective calibration. A step sample with multiple film thickness had been demonstrated to successfully extract the optimal incident angle for maximum phase response.

Chiang and coworkers investigated the SPR sensitivity under various incident wavelengths using SPR polarimetry configuration in recent years. They found that there exists an optimized wavelength with certain film thickness which will deliver the best sensitivity. Conducting experiment at optimized wavelength has led to resolutions of $3.7 \times 10^{-8} \mathrm{RIU}$ for refractive index sensing [47] and $1.9 \times 10^{-6}$ degrees for angle measurement [48] which overmatched most previous works.

Instead of the previously reported direct phase modulation method [37] for SPR phase determination, Sun used an angular modulation method for phase interrogation [49]. A PZT mirror has been employed in a traditional SPR polarimetry setup to introduce an incident angular modulation of $830 \mathrm{~Hz}$ with the amplitude in the order of $10^{-5} \mathrm{rad}$. The signal received in the detector was then modulated by the same frequency. A hybrid parameter relating to both phase and amplitude change is extracted with the help of a lock-in amplifier and subsequently used for SPR sensing. Sensitivity of $5.1 \times 10^{-7} \mathrm{RIU}$ and wide linear measurement range have been reported, and the method is found to be insensitive with varying film thickness for a few nanometers.

In 2009, an interesting research was carried out for spectral phase measurement by a polarimetry setup [50]. In a basic SPR polarimetry setup with the SPR cell and two polaroids as polarizer and analyzer, the laser source was changed to a broadband light source $(1525-1565 \mathrm{~nm})$ and the photodetector was changed to a spectrometer. The spectral interference observed in the spectrometer will be altered when the SPR phase is changed. To facilitate phase measurement, a high-frequency carrier fringe pattern was introduced by incorporating a highly birefringence crystal into the setup which shows different phase retardation for optical waves with different frequencies. Spectral phase was then determined by phase retrieval algorithm. Experiments showed that refractive index change of $8 \times 10^{-6}$ RIU can be easily identified, and a detection limit better than $10^{-6}$ RIU was expected.

SPR imaging based on polarimetry scheme is a promising approach for high throughput sensing. Over 10 years, polarization contrast schemes have been proposed [30, 51] which are found to provide better performance than SPR imaging based on amplitude only [52]. In the polarization contrast scheme, polarizers and waveplates were incorporated into the conventional amplitude-based SPR imaging geometry to create a low-intensity background for uncoated area with bare glass. The sensing areas coated with thin film introduced additional phase changes between the $\mathrm{p}$ - and scomponents, and thus give rise to bright sensing spot which will change its intensity upon changing of SPR coupling

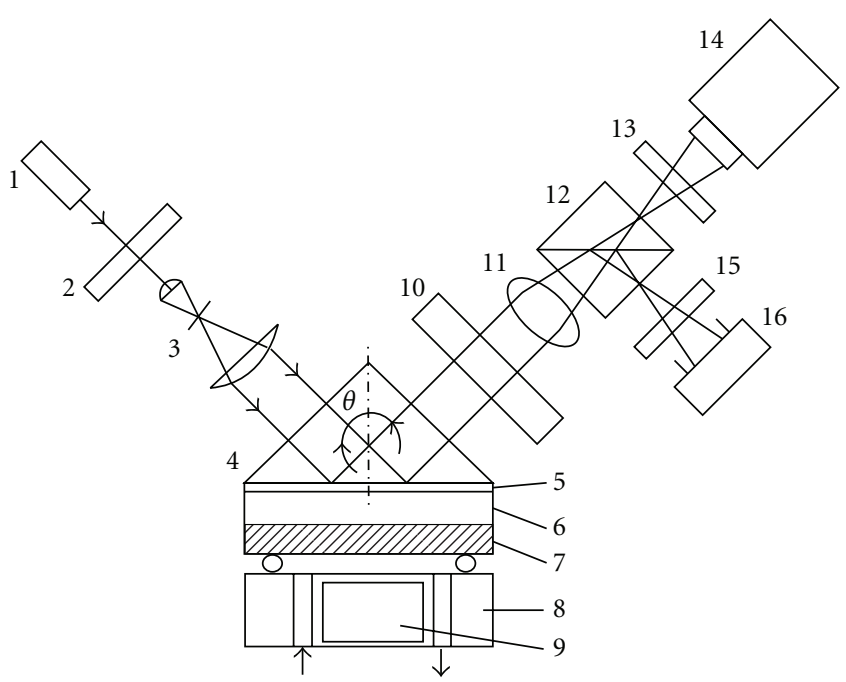

FIGURE 4: Phase shift polarimetry setup for SPR imaging. (1) He-Ne laser; (2) 13, 15, linear polarizers; (3) beam expander with spatial filter; (4) prism; (5) Cr; (6) Au; (7) sample layer; (8) flow cell; (9) TE cooler; (10) liquid-crystal device; (11) image lens; (12) beam splitter; (14) CCD camera; (16) photodiode (Courtesy from [8]).

condition. In their recent works, Homola and coworkers have combined the polarization contrast scheme and specially designed multilayer sensing spots to achieve better sensitivity in parallel sensing [53]. Resolution as low as $2 \times 10^{-6}$ RIU has been achieved in the parallel sensor [54].

Polarization contrast scheme utilizes the SPR phase for more sensitive SPR sensing. However, in the scheme the phase shift amount introduced by the waveplates is fixed. By incorporating variable phase shifting devices for better phase control, the SPR phase can be more accurately determined. In 2005, Su et al. incorporated phase shift interferometry into SPR polarimetry and realized SPR imaging with higher sensitivity [8]. In their proposed setup as shown in Figure 4, a liquid crystal phase retarder is incorporated to introduce a few accurate phase shifts and a two-dimensional CCD camera instead of a point photodetector is used for recording the interference intensities of the $\mathrm{p}$ - and s-components. The SPR-induced phase difference is subsequently determined by a simple 5-step phase shifting algorithm. Owing to the common path feature of polarimetry scheme, phase stability of $2.5 \times 10^{-4} \pi$ was demonstrated and a resolution of $2 \times 10^{-7}$ RIU was reported. Lateral resolution of better than $100 \mu \mathrm{m}$ has been demonstrated in their experiment based on DNA microarray. In 2008, Yu et al. used a similar setup and the Stoilov algorithm for phase retrieval. Resolution of $10^{-6}$ RIU is expected to be achieved based on their SPR imaging experiments on $\mathrm{NaCl}$ concentration and biomolecular interaction [55].

So far, most of the research works reported on SPR polarimetry are based on the Kretschmann-Raether optical platform. Interestingly, a polarization contrast scheme has been recently reported which is based on a gold grating on a commercially available low-cost compact disk [56]. Performance for this system have been demonstrated using helium, air, and carbon dioxide gases, and the SPR responses 
by replacing air with helium or carbon dioxide are clearly identified.

\section{Sensors Based on Interferometry}

As phase information of light cannot be directly recorded by photodetectors, interferometry which compares an unknown light beam with a known reference beam is necessary for phase measurement. The aforementioned optical heterodyne and polarimetry/ellipsometry in Sections 2 and 3 are actually kinds of interferometry, where the interference takes place in frequency domain (optical heterodyne) or between polarized components of a single light beam (polarimetry/ellipsometry). Interferometry in the general sense, which is the interference between two spatially separated light beams (e.g., Newton ring experiment and experiments based on various interferometers), has also been widely applied for SPR phase measurement.

Kabashin and Nikitin pioneered in the exploration of interferometry for SPR phase measurement. They proposed the Mach-Zehnder interferometer and common-path interferometer for phase sensitive SPR sensor development. In their setup based on Mach-Zehnder interferometer [58, 59], a laser beam was divided into a signal arm and a reference arm by a beam splitter. The signal arm was then directed into the SPR cell and underwent phase change for the pcomponent, while the reference arm was reflected by a mirror without phase change. The two beams were then recombined by another beam splitter and the desired phase was determined from the interference patterns. Experiment revealed that the exchanging of $\mathrm{Ar}$ and $\mathrm{N}_{2}$ gases in the SPR chamber results in a phase change of $0.6-0.7 \pi$, which leads to the ultrasensitive detection limit of $4 \times 10^{-8}$ RIU if phase resolution of 0.01 degree is assumed. In that experiment, however, practical resolution is only $10^{-6} \mathrm{RIU}$ due to the imperfect environment control. Later they proposed a semicommon-path interferometer $[60,61]$ where a convergent beam was launched onto the SPR cell and the p- and scomponents of the laser beam were separated by a small angle by a birefringence crystal, interference was then generated between the $\mathrm{p}$ - and s-components with the help of a polarizer. In fact, this setup was very similar to polarimetry except for the use of the birefringence crystal which makes the $\mathrm{p}$ - and s-beams propagate in slightly different directions, thus spatial interference fringes are presented due to the different optical paths travelled by the $\mathrm{p}$ - and s-components. On one hand, these fringes facilitate phase determination by image processing algorithms. On the other hand, the sudden deflection of fringes has helped to experimentally verify the angular dependence and film thickness dependence of SPR phase. Application of this scheme for reaction monitoring between antibodies (rabbit anti-2, 4-d serum) and antigen (2, 4-d pesticide) had also been demonstrated. In the following works, they explored the Heaviside phase jump phenomenon in SPR, where the angular dependence of phase may be subject to a sudden jump under certain conditions, in terms of phase topology [62]. SPR imaging schemes using phase contrast mode or fringe mode were also proposed and compared [30]. Microarrays with spatial resolution of $50 \times 50 \mu \mathrm{m}$ had been demonstrated. It was also demonstrated that phase was more sensitive than amplitude for SPR Imaging.

In 2000, Notcovich et al. used the p-components only and the Mach-Zehnder interferometer for SPR phase imaging [64]. Fourier transform was proposed for fringe analysis and phase determination. They used the setup to monitor helium and argon gas flows in the nitrogen atmosphere, and refractive index resolution at the order of $10^{-6}$ RIU had been obtained.

Since 2002, the application of interferometry for SPR phase determination has been extensively explored by Ho and coworkers [57, 63, 65-71]. In their early work [65, 66], a differential phase measurement scheme was proposed, where two flow cells, one is signal arm with biomolecular interaction and the other is reference without any interaction, were employed and the phase difference between the two channels was used as the signal output. This differential scheme has greatly reduced the environmental fluctuations and improved the signal to noise ratio. The other novel point in their work was the phase measurement metrology. Different from previous interferometry technique [61] using polarizers and birefringence crystal, the proposed scheme used a half waveplate to rotate the p-polarized beam by 90 degrees and make it interference with the s-polarized beam. Phase retrieval was then achieved by using a PZT for phase stepping and monitoring the fringe shift in the interference fringes. Owing to its common-path feature and the differential scheme, the setup was found to be more stable for noise immunity when comparing to the MachZehnder setup by Kabashin and Nikitin [59]. In 2004, they proposed another highly sensitive differential scheme for SPR phase measurement. In that scheme [57], as shown in Figure 5, a Wollaston prism was employed and the pand s-components of the laser beam were separated by a small angle. The two p-beams as well as the two s-beams from the reference and signal arms of the Mach-Zehnder interferometer were then brought to interfere at two photo detectors respectively. As the environmental disturbances are identical to the $\mathrm{p}$ - and s-components in each arm, the differential phase signal between two photo detectors had managed to provide an ultra-sensitive parameter for SPR sensing. Experiment results showed a resolution of $5.5 \times$ $10^{-8}$ RIU based on the assumption of 0.01 degree phase resolution. Biomolecular binding reaction between BSA with its antibody had been demonstrated. In 2007, improvement in terms of sensitivity had been made by incorporating the Michelson interferometer-based double-pass configuration [63] and Fabry-Perot interferometer-based multiple-pass configuration [67]. To compare the sensitivity of the doubleand single-pass configurations, the Michelson interferometer which enables double-pass SPR sensing was connected to the previously reported Mach-Zehnder interferometer which enables single-pass SPR sensing (refer to Figure 6). An additional beamsplitter was used to allow $50 \%$ of the light passing through to the Mach-Zehnder interferometer, while the other 50\% was reflected back and passed through the SPR cell for a second time, which induced an additional phase change between the $\mathrm{p}$ - and s-components and enable 


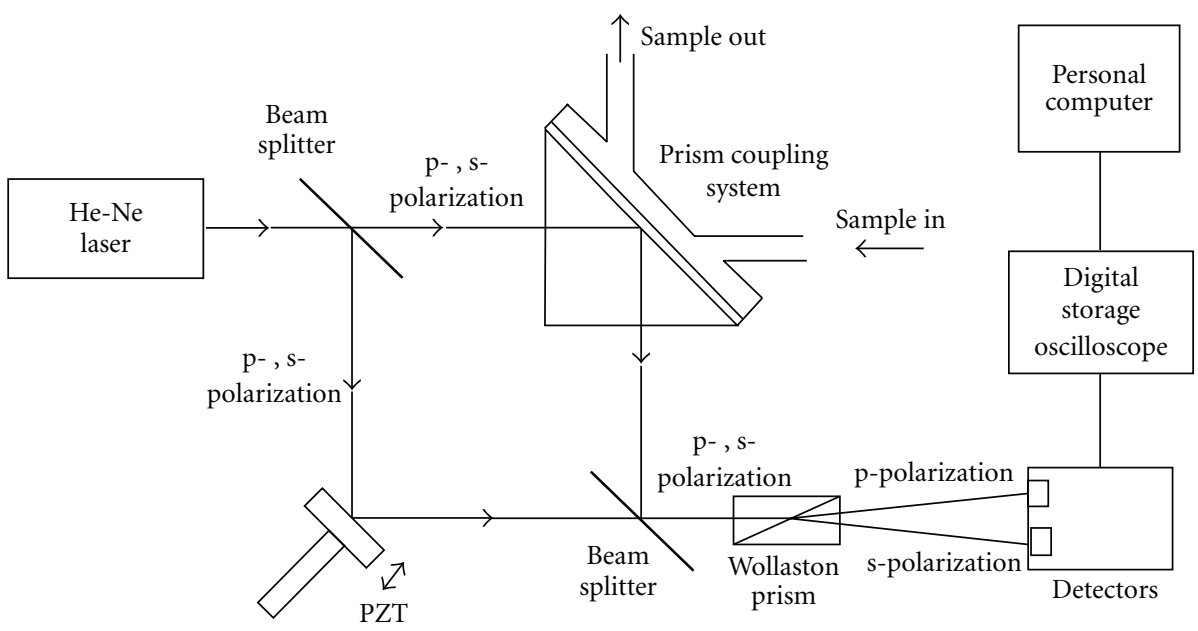

FIGURE 5: Experimental setup for measurement of differential SPR phase shift indicating s and p polarizations on the figure (Courtesy from [57]).

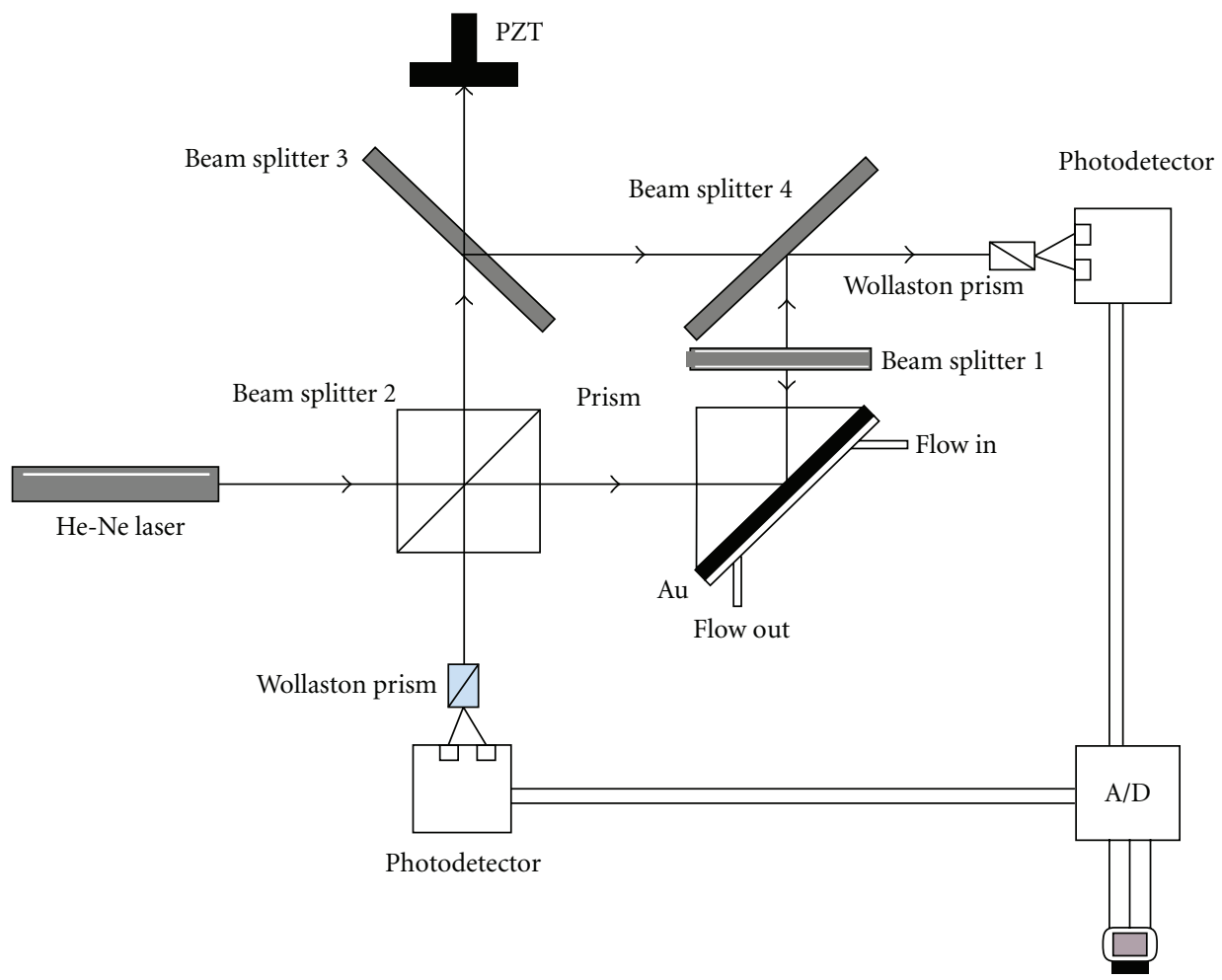

FIGURE 6: Experiment setup for comparing double-pass Michelson interferometer and single pass Mach Zehnder interferometer (Courtesy from [63]).

higher phase response. Calibration experiments of saltwater with different concentrations and biomolecular interaction experiment between BSA and BSA antibody were carried out and it was found that the double-pass signal were almost two times as sensitive as the single-pass signal. Resolution of $7.7 \times 10^{-7} \mathrm{RIU}$ was reported for the double-pass configuration. In the multiple-pass configuration, a FabryPerot interferometer was combined with a beamsplitter, and SPR phase change was accumulated when the laser light was reflected within the Fabry-Perot cavity, while the light intensity was substantially reduced by each pass. The integrated effect was that the multiple-pass interferometer was more sensitive than the double-pass interferometer. The improvement, however, was only $12 \%$ due to the much lower light intensity after multiple attenuated reflections. Recently, SPR imaging for real-time biomolecular interaction sensing was reported based on a polydimethylsiloxane microwell array $[68,69]$ by use of the previous proposed differential 
optical setup [57]. A two-dimensional CMOS camera instead of point photo-detector was employed for interference intensity recording. Phase measurement was fulfilled by pixelwise temporal analysis of the intensity variation curves which was generated by the PZT movement. The proposed phase imaging approach offers a distinct advantage over conventional angular configuration as signals are extracted from temporal sequence and each pixel in the CMOS camera can be a SPR sensor, while angular configuration can at most provide 1D sensing as a one-dimensional array is required to resolve the SPR dip for each sensing element. In addition, the proposed differential phase approach improves detection resolution through removing commonmode disturbances. Experimental results had demonstrated a system resolution of $8.8 \times 10^{-7}$ RIU. Real-time simultaneous monitoring of BSA and anti-BSA binding interactions at various concentration levels had been demonstrated using a single biosensor array. The detection limit of $0.77 \mu \mathrm{g} / \mathrm{mL}$ had been reported. Recently, the high sensitivity feature of phase interrogation and wide dynamic range feature of wavelength interrogation had been successfully combined in a single optical setup $[70,71]$. In the setup, the previously reported double-pass differential scheme [63] using a Michelson interferometer was incorporated for SPR phase measurement with sensitivity enhancement. A second SPR cell without biomolecular interaction was used as a reference cell to provide a reference laser beam for interference. Instead of using laser light, a white light source was used to illuminate the SPR cells and a dual-channel spectrometer was used to resolve the white light interferometry signals for the p- and s-components respectively. From the spectrum of the p-channel signal, the SPR wavelength dip is clearly observed and high frequency oscillations due to white light interferometry were also clearly identified. On the other hand, the spectrum for s-channel represents no apparent SPR dip but only the high frequency oscillation. Spectral phase was determined by transformation technique and the differential phase between $\mathrm{p}$ - and s-components for a specific wavelength was used as the sensor output. When refractive index of the dielectric layer changes due to the biomolecular interaction, the optimal wavelength for maximum sensitivity will shift accordingly, and conventional phase sensitive sensor employing a single wavelength will be subject to lower sensitivity. In this white-light interferometry setup, however, a broadband wavelength was available, thus the wavelength with maximum sensitivity for a particular refractive index could be selected for optimal phase sensing, rendering the method to maintain both high sensitivity and wide dynamic range at the same time. Calibration experiments of saltwater with different concentrations were conducted and a resolution of $2.2 \times 10^{-7} \mathrm{RIU}$ was reported. Biomolecular binding experiment between BSA and BSA antibody were also carried out and the detection limit was estimated as $0.5 \mathrm{ng} / \mathrm{mL}$.

Another SPR imaging system based on phase shift interferometry was reported in 2005 [72]. The optical setup was similar to that from Ho et al. [65] and a half waveplate was used to rotate the s-component by 90 degrees to make it interfere with the $\mathrm{p}$-component. Phase retrieval was enabled by a PZT with 5-step phase shifting algorithm. Realtime experiment for DNA microarray analysis had been conducted and resulted in valuable quantitative information. The phase shifting SPR imaging system offers high resolution and high-throughout biosensing without additional labeling. As a result, a detection limit of $2.5 \times 10^{-7} \mathrm{RIU}$, a long-term phase stability of $\pi / 100$ in 30 minutes, and a spatial resolution of $100 \times 100 \mu \mathrm{m}^{2}$ was achieved. Label-free observation of 15-mer DNA microarray was also demonstrated.

Interestingly, SPR phase had been incorporated into integrated optical sensor composed of fiber optics waveguide for higher sensitivity refractive index sensing $[73,74]$. Both works were based on the Mach-Zehnder interferometer for phase determination. The latter work [74] included a Bragg or long-period grating in the sensing arm for sensitivity enhancement. Both works are based on theoretical simulation for design optimization, and experimental realization of such sensors was ongoing.

\section{Conclusions}

The past decades have envisioned the rapid development of SPR sensors both in optical readout unit and biorecognition coating. Because of the ease of assay preparation without the need of any labeling, SPR sensors have become a standard tool in various biological, medical, and chemical applications. In this paper, various phase detection schemes have been summarized and their potential improvements in terms of sensitivity and throughput have been discussed. We envision that SPR sensors based on temporal phase modulation will serve as high throughput label-free biosensor for next generation.

\section{Acknowledgments}

The authors wish to acknowledge financial support from a General Research Grant, Hong Kong Research Grants Council, under Project nos. 413008 and 411208, and a Group Research Project (no. 3110048) from Chinese University of Hong Kong.

\section{References}

[1] R. W. Wood, "XLII. On a remarkable case of uneven distribution of light in a diffraction grating spectrum," Philosophical Magazine Series 6, vol. 4, no. 21, p. 396, 1902.

[2] J. Zenneck, "Über die Fortpflanzung ebener elektromagnetischer Wellen längs einer ebenen Leiterfläche und ihre Beziehung zur drahtlosen telegraphie," Annalen der Physik, vol. 328, no. 10, pp. 846-866, 1907.

[3] A. Otto, "Excitation of nonradiative surface plasma waves in silver by the method of frustrated total reflection," Zeitschrift für Physik, vol. 216, no. 4, pp. 398-410, 1968.

[4] E. Kretschmann and H. Z. Raether, "Radiative decay of non-radiative surface plasmons excited by light," Verlag der Zeitschrift für Naturforschung, vol. 23, pp. 2135-2136, 1968.

[5] F. Abeles, "Surface electromagnetic waves ellipsometry," Surface Science, vol. 56, pp. 237-251, 1976. 
[6] A. V. Kabashin, S. Patskovsky, and A. N. Grigorenko, "Phase and amplitude sensitivities in surface plasmon resonance bio and chemical sensing," Optics Express, vol. 17, no. 23, pp. 21191-21204, 2009.

[7] X. L. Yu, D. X. Wang, X. Wei, D. Xiang, L. Wei, and Z. Xinsheng, "A surface plasmon resonance imaging interferometry for protein micro-array detection," Sensors and Actuators B, vol. 108, no. 1-2, pp. 765-771, 2005.

[8] Y. D. Su, S. J. Chen, and T. L. Yeh, "Common-path phase-shift interferometry surface plasmon resonance imaging system," Optics Letters, vol. 30, no. 12, pp. 1488-1490, 2005.

[9] J. Homola, S. S. Yee, and G. Gauglitz, "Surface plasmon resonance sensors: review," Sensors and Actuators B, vol. 54, no. 1-2, pp. 3-15, 1999.

[10] C. Boozer, G. Kim, S. Cong, H. Guan, and T. Londergan, "Looking towards label-free biomolecular interaction analysis in a high-throughput format: a review of new surface plasmon resonance technologies," Current Opinion in Biotechnology, vol. 17, no. 4, pp. 400-405, 2006.

[11] K. S. Phillips and Q. Cheng, "Recent advances in surface plasmon resonance based techniques for bioanalysis," Analytical and Bioanalytical Chemistry, vol. 387, no. 5, pp. 1831-1840, 2007.

[12] J. Homola, "Surface plasmon resonance sensors for detection of chemical and biological species," Chemical Reviews, vol. 108, no. 2, pp. 462-493, 2008.

[13] J. Homola, Ed., Surface Plasmon Resonance Based Sensors, Springer, Berlin, Germany, 2006.

[14] R. B. M. Schasfoort and A. J. Tudos, Eds., Handbook of Surface Plasmon Resonance, Royal Society of Chemistry, Cambridge, UK, 2008.

[15] S. G. Nelson, K. S. Johnston, and S. S. Yee, "High sensitivity surface plasmon resonance sensor based on phase detection," Sensors and Actuators B, vol. 35-36, no. 1-3, pp. 187-191, 1996.

[16] S. Shen, T. Liu, and J. Guo, "Optical phase-shift detection of surface plasmon resonance," Applied Optics, vol. 37, no. 10, pp. 1747-1751, 1998.

[17] Y. Xinglong, Z. Lequn, J. Hong, W. Haojuan, Y. Chunyong, and Z. Shenggeng, "Immunosensor based on optical heterodyne phase detection," Sensors and Actuators B, vol. 76, no. 1-3, pp. 199-202, 2001.

[18] C. M. Wu, Z. C. Jian, S. F. Joe, and L. B. Chang, "Highsensitivity sensor based on surface plasmon resonance and heterodyne interferometry," Sensors and Actuators B, vol. 92, no. 1-2, pp. 133-136, 2003.

[19] M. H. Chiu, S. F. Wang, and R. S. Chang, "D-type fiber biosensor based on surface-plasmon resonance technology and heterodyne interferometry," Optics Letters, vol. 30, no. 3, pp. 233-235, 2005.

[20] S. F. Wang, M. H. Chiu, and R. S. Chang, "Numerical simulation of a D-type optical fiber sensor based on the Kretchmann's configuration and heterodyne interferometry," Sensors and Actuators B, vol. 114, no. 1, pp. 120-126, 2006.

[21] M. H. Chiu and C. H. Shih, "Searching for optimal sensitivity of single-mode D-type optical fiber sensor in the phase measurement," Sensors and Actuators B, vol. 131, no. 2, pp. 596-601, 2008.

[22] S. F. Wang, "U-shaped optical fiber sensor based on multiple total internal reflections in heterodyne interferometry," Optics and Lasers in Engineering, vol. 47, no. 10, pp. 1039-1043, 2009.
[23] Y. C. Li, Y. F. Chang, L. C. Su, and C. Chou, "Differential-phase surface plasmon resonance biosensor," Analytical Chemistry, vol. 80, no. 14, pp. 5590-5595, 2008.

[24] W. C. Kuo, C. Chou, and H. T. Wu, "Optical heterodyne surface-plasmon resonance biosensor," Optics Letters, vol. 28, no. 15, pp. 1329-1331, 2003.

[25] C. Chou, H. T. Wu, Y. C. Huang, Y. L. Chen, and W. C. Kuo, "Characteristics of a paired surface plasma waves biosensor," Optics Express, vol. 14, no. 10, pp. 4307-4315, 2006.

[26] S. Herminghaus, C. Bechinger, W. Petersen, and P. Leiderer, "Phase contrast surface mode resonance microscopy," Optics Communications, vol. 112, no. 1-2, pp. 16-20, 1994.

[27] A. A. Kruchinin and Y. G. Vlasov, "Surface plasmon resonance monitoring by means of polarization state measurement in reflected light as the basis of a DNA-probe biosensor," Sensors and Actuators B, vol. 30, no. 1, pp. 77-80, 1996.

[28] V. E. Kochergin, A. A. Beloglazov, M. V. Valeiko, and P. I. Nikitin, "Phase properties of a surface-plasmon resonance from the viewpoint of sensor applications," Quantum Electronics, vol. 28, no. 5, pp. 444-448, 1998.

[29] A. V. Kabashin, V. E. Kochergin, A. A. Beloglazov, and P. I. Nikitin, "Phase-polarisation contrast for surface plasmon resonance biosensors," Biosensors and Bioelectronics, vol. 13, no. 12, pp. 1263-1269, 1998.

[30] P. I. Nikitin, A. N. Grigorenko, A. A. Beloglazov et al., "Surface plasmon resonance interferometry for micro-array biosensing," Sensors and Actuators A, vol. 85, no. 1, pp. 189193, 2000.

[31] P. Westphal and A. Bornmann, "Biomolecular detection by surface plasmon enhanced ellipsometry," Sensors and Actuators B, vol. 84, no. 2-3, pp. 278-282, 2002.

[32] M. Poksinski and H. Arwin, "In situ monitoring of metal surfaces exposed to milk using total internal reflection ellipsometry," Sensors and Actuators B, vol. 94, no. 3, pp. 247-252, 2003.

[33] R. Naraoka and K. Kajikawa, "Phase detection of surface plasmon resonance using rotating analyzer method," Sensors and Actuators B, vol. 107, no. 2, pp. 952-956, 2005.

[34] I. R. Hooper and J. R. Sambles, "Sensing using differential surface plasmon ellipsometry," Journal of Applied Physics, vol. 96, no. 5, pp. 3004-3011, 2004.

[35] I. R. Hooper and J. R. Sambles, "Differential ellipsometric surface plasmon resonance sensors with liquid crystal polarization modulators," Applied Physics Letters, vol. 85, no. 15, pp. 3017-3019, 2004.

[36] I. R. Hooper, J. R. Sambles, M. C. Pitter, and M. G. Somekh, "Phase sensitive array detection with polarisation modulated differential sensing," Sensors and Actuators B, vol. 119, no. 2, pp. 651-655, 2006.

[37] H. P. Ho, W. C. Law, S. Y. Wu et al., "Phase-sensitive surface plasmon resonance biosensor using the photoelastic modulation technique," Sensors and Actuators B, vol. 114, no. 1, pp. 80-84, 2006.

[38] H. J. Peng, S. P. Wong, Y. W. Lai, X. H. Liu, H. P. Ho, and S. Zhao, "Simplified system based on photoelastic modulation technique for low-level birefringence measurement," Review of Scientific Instruments, vol. 74, no. 11, pp. 4745-4749, 2003.

[39] W. Yuan, H. P. Ho, S. Y. Wu, Y. K. Suen, and S. K. Kong, "Polarization-sensitive surface plasmon enhanced ellipsometry biosensor using the photoelastic modulation technique," Sensors and Actuators A, vol. 151, no. 1, pp. 23-28, 2009. 
[40] C. E. Stewart, I. R. Hooper, and J. R. Sambles, "Surface plasmon differential ellipsometry of aqueous solutions for bio-chemical sensing," Journal of Physics D, vol. 41, no. 10, pp. 105408-105415, 2008.

[41] I. R. Hooper, M. Rooth, and J. R. Sambles, "Dual-channel differential surface plasmon ellipsometry for bio-chemical sensing," Biosensors and Bioelectronics, vol. 25, no. 2, pp. 411417, 2009.

[42] P. P. Markowicz, W. C. Law, A. Baev, P. N. Prasad, S. Patskovsky, and A. V. Kabashin, "Phase-sensitive time-modulated surface plasmon resonance polarimetry for wide dynamic range biosensing," Optics Express, vol. 15, no. 4, pp. 17451754, 2007.

[43] W. C. Law, P. Markowicz, K. T. Yong et al., "Wide dynamic range phase-sensitive surface plasmon resonance biosensor based on measuring the modulation harmonics," Biosensors and Bioelectronics, vol. 23, no. 5, pp. 627-632, 2007.

[44] S. Patskovsky, R. Jacquemart, M. Meunier, G. de Crescenzo, and A. V. Kabashin, "Phase-sensitive spatially-modulated surface plasmon resonance polarimetry for detection of biomolecular interactions," Sensors and Actuators B, vol. 133, no. 2, pp. 628-631, 2008.

[45] S. Patskovsky, M. Maisonneuve, M. Meunier, and A. V. Kabashin, "Mechanical modulation method for ultra-sensitive phase measurements in photonics biosensing," Optics Express, vol. 16, no. 26, pp. 21305-21314, 2008.

[46] S. Patskovsky, M. Vallieres, M. Maisonneuve, I. H. Song, M. Meunier, and A. V. Kabashin, "Designing efficient zero calibration point for phase-sensitive surface plasmon resonance biosensing," Optics Express, vol. 17, no. 4, pp. 2255-2263, 2009.

[47] H. P. Chiang, J. L. Lin, and Z. W. Chen, "High sensitivity surface plasmon resonance sensor based on phase interrogation at optimal incident wavelengths," Applied Physics Letters, vol. 88, no. 14, Article ID 141105, 2006.

[48] H. P. Chiang, J. L. Lin, R. Chang, S. Y. Su, and P. T. Leung, "High-resolution angular measurement using surfaceplasmon-resonance via phase interrogation at optimal incident wavelengths," Optics Letters, vol. 30, no. 20, pp. 2727 2729, 2005.

[49] Z. Sun, Y. He, and J. Guo, "Surface plasmon resonance sensor based on polarization interferometry and angle modulation," Applied Optics, vol. 45, no. 13, pp. 3071-3076, 2006.

[50] Z. Zheng, Y. Wan, X. Zhao, and J. Zhu, "Spectral interferometric measurement of wavelength-dependent phase response for surface plasmon resonance sensors," Applied Optics, vol. 48, no. 13, pp. 2491-2495, 2009.

[51] J. Homola and S. S. Yee, "Novel polarization control scheme for spectral surface plasmon resonance sensors," Sensors and Actuators B, vol. 51, no. 1-3, pp. 331-339, 1998.

[52] G. Steiner, V. Sablinskas, A. Hübner, C. Kuhne, and R. Salzer, "Surface plasmon resonance imaging of microstructured monolayers," Journal of Molecular Structure, vol. 509, no. 1-3, pp. 265-273, 1999.

[53] M. Piliarik, H. Vaisocherová, and J. Homola, "A new surface plasmon resonance sensor for high-throughput screening applications," Biosensors and Bioelectronics, vol. 20, no. 10, pp. 2104-2110, 2005.

[54] M. Piliarik, H. Vaisocherová, and J. Homola, "Towards parallelized surface plasmon resonance sensor platform for sensitive detection of oligonucleotides," Sensors and Actuators $B$, vol. 121, no. 1, pp. 187-193, 2007.

[55] X. Yu, X. Ding, F. Liu, and Y. Deng, "A novel surface plasmon resonance imaging interferometry for protein array detection," Sensors and Actuators B, vol. 130, no. 1, pp. 52-58, 2008.
[56] N. Sedoglavich, R. Künnemeyer, S. R. Talele, and J. C. Sharpe, "Phase-polarisation contrast for surface plasmon resonance based on low cost grating substrates," Current Applied Physics, vol. 8, no. 3-4, pp. 351-354, 2008.

[57] S. Y. Wu, H. P. Ho, W. C. Law, and C. Lin, "Highly sensitive differential phase-sensitive surface plasmon resonance biosensor based on the Mach-Zehnder configuration," Optics Letters, vol. 29, no. 20, pp. 2378-2380, 2004.

[58] A. V. Kabashin and P. I. Nikitin, "Interferometer based on a surface plasmon resonance for sensor applications," Quantum Electronics, vol. 27, no. 7, pp. 653-654, 1997.

[59] A. V. Kabashin and P. I. Nikitin, "Surface plasmon resonance interferometer for bio- and chemical-sensors," Optics Communications, vol. 150, no. 1-6, pp. 5-8, 1998.

[60] V. E. Kochergin, M. V. Valeiko, A. A. Beloglazov, T. I. Ksenevich, and P. I. Nikitin, "Visualisation of the angular dependence of the reflected-radiation phase under conditions of a surface-plasmon resonance and its sensor applications," Quantum Electronics, vol. 28, no. 9, pp. 835-839, 1998.

[61] P. I. Nikitin, A. A. Beloglazov, V. E. Kochergin, M. V. Valeiko, and T. I. Ksenevich, "Surface plasmon resonance interferometry for biological and chemical sensing," Sensors and Actuators B, vol. 54, no. 1, pp. 43-50, 1999.

[62] A. N. Grigorenko, P. I. Nikitin, and A. V. Kabashin, "Phase jumps and interferometric surface plasmon resonance imaging," Applied Physics Letters, vol. 75, no. 25, pp. 3917-3919, 1999.

[63] W. Yuan, H. P. Ho, C. L. Wong, S. K. Kong, and C. Lin, "Surface plasmon resonance biosensor incorporated in a michelson interferometer with enhanced sensitivity," IEEE Sensors Journal, vol. 7, no. 1, pp. 70-73, 2007.

[64] A. G. Notcovich, V. Zhuk, and S. G. Lipson, "Surface plasmon resonance phase imaging," Applied Physics Letters, vol. 76, no. 13 , pp. 1665-1667, 2000.

[65] H. P. Ho, W. W. Lam, and S. Y. Wu, "Surface plasmon resonance sensor based on the measurement of differential phase," Review of Scientific Instruments, vol. 73, no. 10, pp. 3534-3539, 2002.

[66] H. P. Ho and W. W. Lam, "Application of differential phase measurement technique to surface plasmon resonance sensors," Sensors and Actuators B, vol. 96, no. 3, pp. 554-559, 2003.

[67] H. P. Ho, W. Yuan, C. L. Wong et al., "Sensitivity enhancement based on application of multi-pass interferometry in phasesensitive surface plasmon resonance biosensor," Optics Communications, vol. 275, no. 2, pp. 491-496, 2007.

[68] C. L. Wong, H. P. Ho, T. T. Yu et al., "Two-dimensional biosensor arrays based on surface plasmon resonance phase imaging," Applied Optics, vol. 46, no. 12, pp. 2325-2332, 2007.

[69] C. L. Wong, H. P. Ho, Y. K. Suen et al., "Real-time protein biosensor arrays based on surface plasmon resonance differential phase imaging," Biosensors and Bioelectronics, vol. 24, no. 4, pp. 606-612, 2008.

[70] S. P. Ng, C. M. L. Wu, S. Y. Wu, H. P. Ho, and S. K. Kong, "Differential spectral phase interferometry for wide dynamic range surface plasmon resonance biosensing," Biosensors and Bioelectronics, vol. 26, no. 4, pp. 1593-1598, 2010.

[71] S. P. Ng, C. M. L. Wu, S. Y. Wu, and H. P. Ho, "White-light spectral interferometry for surface plasmon resonance sensing applications," Optics Express, vol. 19, no. 5, pp. 4521-4527, 2011. 
[72] S. J. Chen, Y. D. Su, F. M. Hsiu, C. Y. Tsou, and Y. K. Chen, "Surface plasmon resonance phase-shift interferometry: real-time DNA microarray hybridization analysis," Journal of Biomedical Optics, vol. 10, no. 3, Article ID 034005, 2005.

[73] A. K. Sheridan, R. D. Harris, P. N. Bartlett, and J. S. Wilkinson, "Phase interrogation of an integrated optical SPR sensor," Sensors and Actuators B, vol. 97, no. 1, pp. 114-121, 2004.

[74] G. Nemova, A. V. Kabashin, and R. Kashyap, "Surface plasmon-polariton Mach-Zehnder refractive index sensor," Journal of the Optical Society of America B, vol. 25, no. 10, pp. 1673-1677, 2008. 

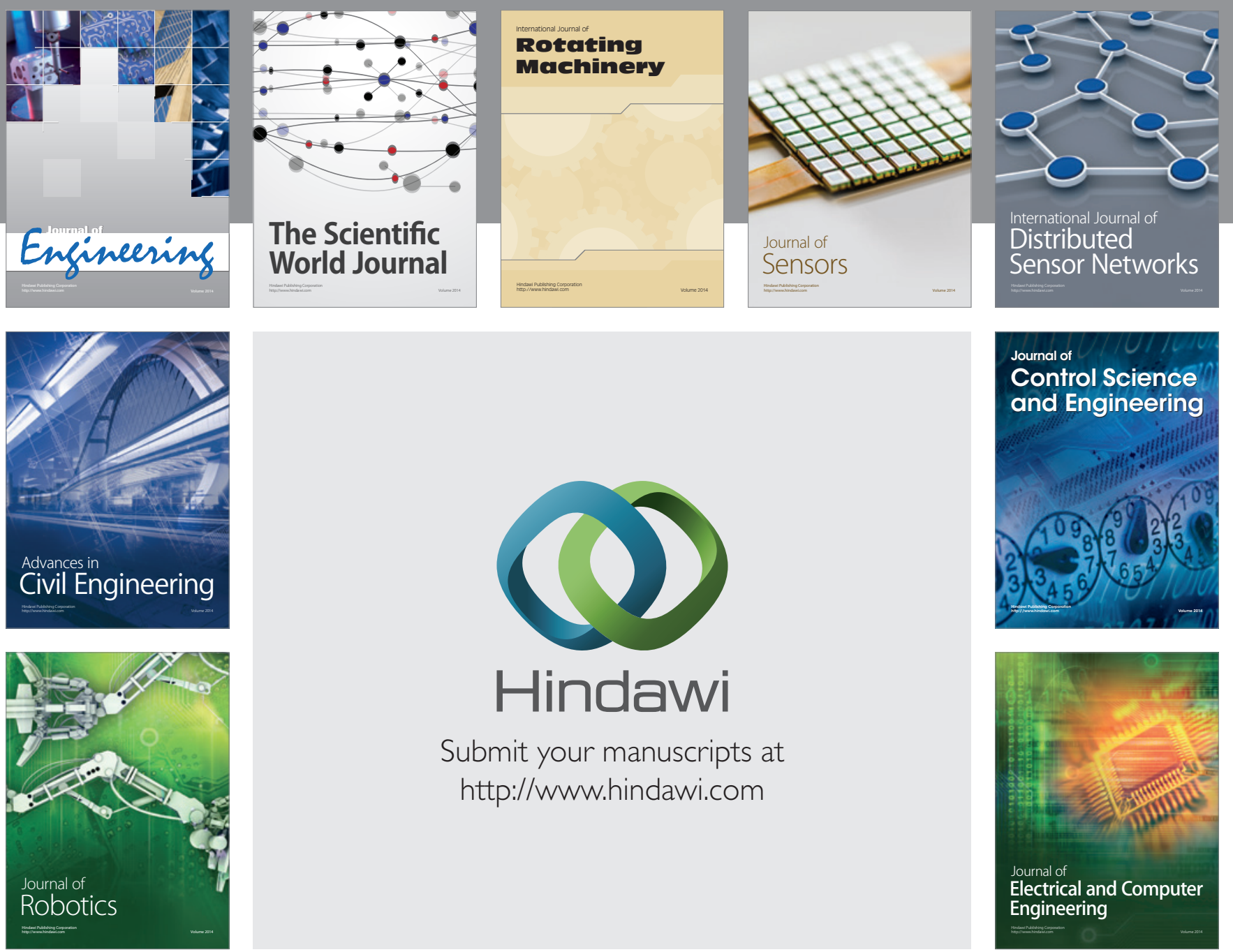

Submit your manuscripts at

http://www.hindawi.com
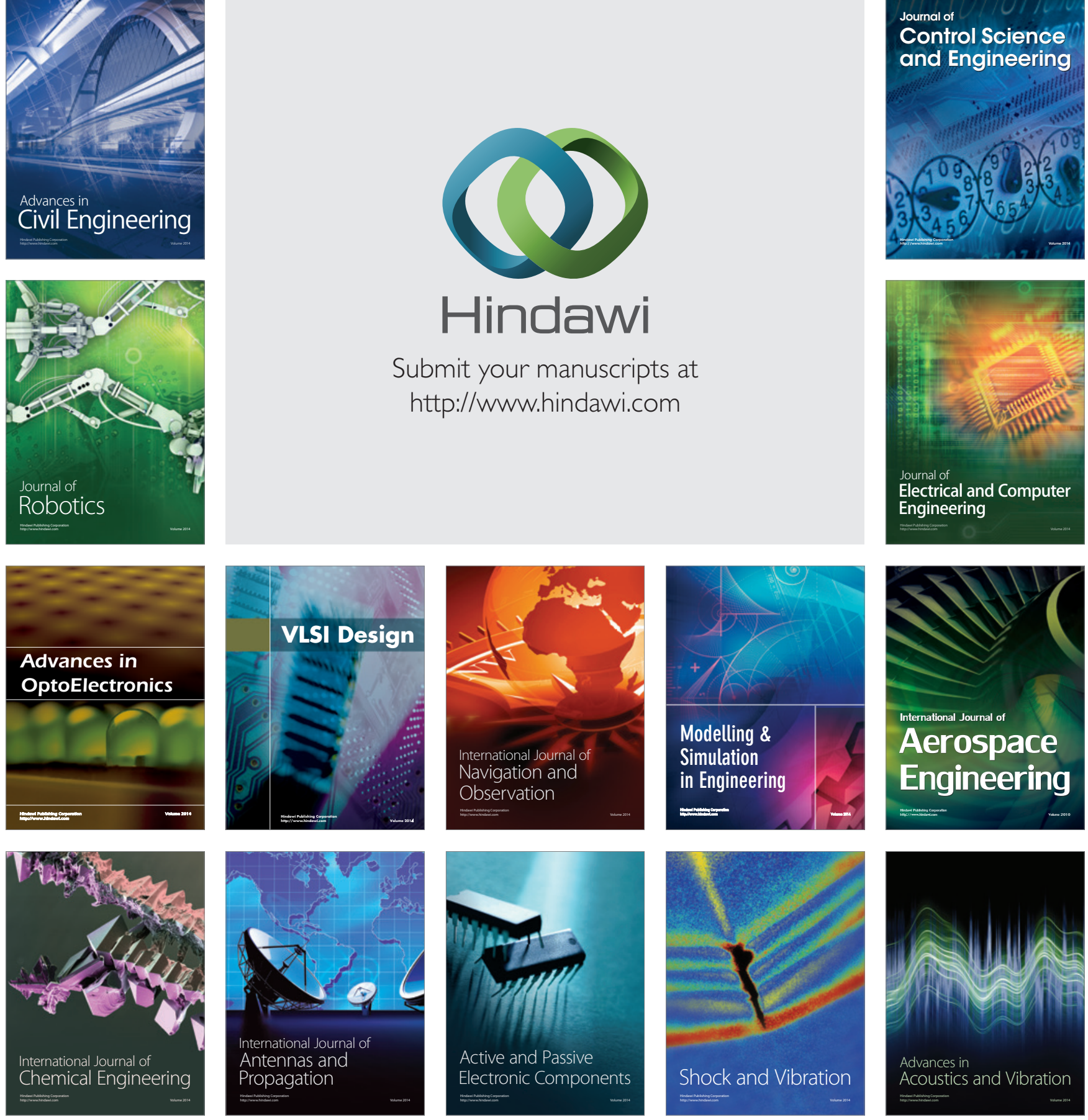